\title{
A novel analytic measure for the water maze utilizing the concept of entropy
}

\author{
Hye-Ryeon Lee ${ }^{1}$ and Bong-Kiun Kaang ${ }^{1,2 *}$ \\ 1 Department of Biological Sciences, Seoul National University, Seoul, Korea \\ 2 Department of Brain and Cognitive Sciences, Seoul National University, Seoul, Korea \\ ${ }^{*}$ Correspondence: kaang@snu.ac.kr
}

\section{A commentary on}

What is the most sensitive measure of water maze probe test performance?

by Hamid R. Maei, Kirill Zaslavsky, Afra $H$. Wang, Adelaide P. Yiu, Catia M. Teizeira, Sheena A. Josselyn and Paul W. Frankland

The Morris water maze, a spatial learning task first introduced by Dr. Richard Morris (Morris, 1981, 1984), is one of the most extensively used behavioral paradigms used to examine hippocampus-dependent spatial learning and memory in rodents, such as rats and mice (Tanda et al., 2009). In water maze tests, animals are forced to swim in a circular pool of milky water. In order to escape from the water, the animals are required to locate a submerged hidden platform with the aid of spatial cues surrounding the water pool. Once practiced, the animals begin to learn the relationship between the platform location and various spatial cues, and eventually form a memory of the location of the hidden platform (Morris, 1984). To determine whether animals acquire a spatial learning about the location of the hidden platform and are able to search for the platform solely by relying on spatial memory, probe trials are performed where the escape platform is removed from the pool and the animals are permitted to swim and search the pool for $1 \mathrm{~min}$. In the probe trial, the occupational time spent in the specific quadrant where the platform was originally placed and the number of crossings through the former hidden platform location are used as general measures to quantify the level of spatial memory.

Since its development, the water maze has become the core assay of behavioral test batteries used to analyze hippocampus-dependent brain functions, and has subsequently been exploited to explore the functions of other areas of the brain (Tsien et al., 1996; D’Hooge and De Deyn, 2001). Moreover, a variety of protocols, including reversal training and delayed matching-toplace paradigm, have been established for investigating the role of specific genes or proteins in normal and abnormal brain functions (Tsien et al., 1996; Gallagher and Rapp, 1997; Lipp and Wolfer, 1998; Vorhees and Williams, 2006). In contrast to this progress, however, few studies have questioned the effectiveness of the existing analytic measures, such as percentage quadrant time, percentage zone, and platform crossings, that are used in water maze studies. Furthermore, relatively few attempts have been made to improve the sensitivity of the water maze in order to detect possible subtle phenotypic changes that might occur between groups during learning (Maei et al., 2009).

In a research article published in Frontiers in Neuroscience, Maei et al. (2009) describe a newly developed analytical method for detecting group differences more sensitively and accurately in the water maze. Herein, the authors introduced the concept of entropy $(\mathrm{H})$ a measure of the disorder of a system. The rationale underlying this idea is that over the course of water maze training, an animal's search strategy might be shifted from disorganized to more focal searching (Vorhees and Williams, 2006), which can be considered as a reduction of disorder in the system. By using entropy $(\mathrm{H})$ as an analytical measure for the water maze, Maei et al. (2009) argue that we can more fully exploit an animal's positional data produced by tracking software, thereby providing greater sensitivity for detecting phenotypic differences.

To evaluate how $\mathrm{H}$ works and to compare the usefulness of $\mathrm{H}$ with existing measures, the authors rendered a series of Monte Carlo simulations and randomly selected individual trials from a dataset of more than 1600 probe tests. This approach allowed them to simulate water maze experiments with varying sample and effect sizes. Next, by summing two types of variance - error variance (the variance of an animal's position with respect to the target; $\mathrm{H}_{\text {error }}$ ) and path variance (the variance of an animal's position with respect to its path; $\mathrm{H}_{\text {path }}$ ) - and using the summed value as a measure of the entropy of spatial navigation, $\mathrm{H}$ could thoroughly utilize the positional information from each tracking record. Lastly, they experimentally verified $\mathrm{H}$ using three paradigms of hippocampal dysfunctions.

The authors report that $\mathrm{H}$ outperforms existing measures in terms of its sensitivity in detecting group differences over a range of sample or effect sizes. As discussed by the authors, the excellence of $\mathrm{H}$ can be recapitulated by two points. One concerns its full usage of precise positional information of an animal throughout the probe test. Represented by $\mathrm{H}_{\text {error }}$ and $\mathrm{H}_{\text {path }}$, the $\mathrm{H}$ measure assesses both the degree to which searching is focused on the former platform location and how focused the search is. Using this approach, $\mathrm{H}$ can hold a greater amount of detailed trajectory information than any other measure, which confers exceptional sensitivity upon $\mathrm{H}$, particularly for comparisons between subjects. In addition, by adjusting the relative weight between $\mathrm{H}_{\text {error }}$ and $\mathrm{H}_{\text {path' }}$, $\mathrm{H}$ can be adjusted to outperform other measures under various experimental conditions. The second point is that the $\mathrm{H}$ measure turns out to be distributed normally, which is in stark contrast to other measures (Maei et al., 2009). This characteristic gives a further advantage to the $\mathrm{H}$ measure because normality is a prerequisite for parametric tests, such as the t-test and ANOVA, to yield correct statistical results without type-1 errors. Moreover, parametric tests are the most widely used statistical methods for analyzing behavioral test batteries. 
To summarize, the analytical approach described by Maei et al. (2009) provides a more effective and accurate new measure in terms of its superb sensitivity and statistical validity in water maze studies. Furthermore, the $\mathrm{H}$ measure will contribute greatly to the advancement of behavioral assays that, in conjunction with molecular genetic approaches, are designed to elucidate the molecular mechanisms underlying learning and memory.

\section{REFERENCES}

D'Hooge, R., and De Deyn, P. P. (2001). Applications of the Morris water maze in the study of learning and memory. Brain Res. Brain Res. Rev. 36, 60-90.
Gallagher, M., and Rapp, P. R. (1997). The use of animal models to study the effects of aging on cognition. Annu. Rev. Psychol. 48, 339-370.

Lipp, H. P., and Wolfer, D. P. (1998). Genetically modified mice and cognition. Curr. Opin. Neurobiol. 8, 272-280.

Maei, H. R., Zaslavsky, K., Teixeira, C. M., and Frankland, P. W. (2009). What is the most sensitive measure of water maze probe test performance? Front. Integr. Neurosci. 3, 4. doi: 10.3389/neuro.07.004.2009.

Morris, R.G.M.(1981).Spatial localization does not require the presence of local cues. Learn. Motiv. 12, 239-260.

Morris, R. G. M. (1984). Developments of a water-maze procedure for studying spatial learning in the rat. $J$. Neurosci. Methods 11, 47-60.

Tanda, K., Nishi, A., Matsuo, N., Nakanishi, K., Yamasaki, N., Sugimoto, T., Toyama, K., Takao, K., and Miyakawa, T. (2009). Abnormal social behavior, hyperactivity, impaired remote spatial memory, and increased D1-mediated dopaminergic signaling in neuronal nitric oxide synthase knockout mice. $M o l$. Brain 2, 19.

Tsien, J. Z., Huerta, P. T., and Tonegawa, S. (1996). The essential role of hippocampal CA1 NMDA receptordependent synaptic plasticity in spatial memory. Cell 87, 1327-1338.

Vorhees, C. V., and Williams, M. T. (2006). Morris water maze: procedures for assessing spatial and related forms of learning and memory. Nat. Protoc. 1, 848-858.

Received: 21 September 2009; published: 15 April 2010. Citation: Front. Neurosci. (2010) 4, 1: 1-2. doi: 10.3389/ neuro.01.002.2010

Copyright $\odot 2010$ Lee and Kaang. This is an open-access publication subject to an exclusive license agreement between the authors and the Frontiers Research Foundation, which permits unrestricted use, distribution, and reproduction in any medium, provided the original authors and source are credited.

\section{Decisions of voluntary action: what vs when}

\section{Deborah J. Serrien* \\ School of Psychology, University of Nottingham, University Park, Nottingham, UK \\ *Correspondence: deborah.serrien@nottingham.ac.uk}

\section{A commentary on}

\section{Dissociating what and when of intentional actions}

by Veronika Krieghoff, Marcel Brass, Wolfgang Prinz and Florian Waszak

Voluntary action is a fundamental characteristic of human behavior and identifies our ability to realize intentionally driven tasks. Functional imaging work has indicated that intentional performance activates neural circuitry confined to the fronto-medial cortex (Cunnington et al., 2002; Lau et al., 2004; Nachev et al., 2005). This network activity integrates, besides the conscious experience of intending (Haggard and Clark, 2003; Lau et al., 2004), a series of processes in relation to the intentional act (Brass and Haggard, 2007). Accordingly, intentional behavior can be viewed as a form of decision making that consists of various components with different types of information processing (Brass and Haggard, 2008; Haggard, 2008). This distinctive viewpoint is important as voluntary action has often been regarded as a unitary concept without little con- sideration for the component functions. In this respect, it has been suggested that voluntary action has at least two decisional processes: what action to perform (selection component) and when to perform it (timing component), (Mueller et al., 2007). Thus, the "what" decision specifies which action to perform from a range of alternative options, whereas the "when" decision denotes the moment in time at which to execute the action.

In their recent contribution to Frontiers in Neuroscience, Krieghoff et al. (2009) presented an fMRI study that provides new insights into voluntary behavior. In particular, the authors proposed a welldesigned protocol in which they simultaneously and independently manipulated the selection and timing component within one experimental paradigm. Moreover, the participants were instructed to perform one of two possible actions at one of two possible moments in time. Furthermore, the action and moment in time were freely chosen or externally triggered by means of a cue. In other words, the "what" (selection) and "when" (timing) decision of the impending action were based on an internal judgment or imposed by an external stimulus. This distinction between internally and exter- nally generated responses is relevant as both types of activities are known to have (partly) dissociable neural circuitry in respect to the component process of selection, as well as timing (Deiber et al., 1999; Cunnington et al., 2002, 2006; Lau et al., 2006).

As the main objective of Krieghoff et al. (2009) was to investigate the decisional processes of voluntary behavior, the data analysis focused primarily on the cue-related activity that specified the decision making process rather than on the target-related activity that represented the implementation of that decision. In view of that premise, the whole-brain analysis of the cue-related signal showed that two fronto-medial wall areas associated with distinct component functions of voluntary action: whereas the selection component linked strongly with the rostral cingulate zone (RCZ), the timing component tied closely with the superior medial frontal gyrus (SFG), which is localized close to pre-SMA (Rushworth, 2008). These results indicate that separate brain regions are involved in distinct decisional processes, and accordingly highlight a functional dissociation of intentional action. However, an additional 